\title{
A GENERALIZATION OF THE GAUSS-BONNET THEOREM
}

\author{
BY \\ JAMES EELLS, JR.(1)
}

1. Introduction. In this paper we are concerned with relations between the characteristic classes and the curvature of a Riemann manifold $X$. Our main result (Theorem 4D) establishes a representation formula for the StiefelWhitney classes of $X$ in terms of integrals of certain generalized Gauss and geodesic curvature forms on $X$.

(A) The Stiefel-Whitney classes $w^{r}(X)$ are cohomology classes of $X$ of order 2 for $1 \leqq r<n=\operatorname{dim} X$. That these classes admit an integral representation formula is assured by the following existence theorem of AllendoerferEells [3], which we state here for future reference.

A $(\boldsymbol{Z}, \boldsymbol{r})$-pair $(\boldsymbol{Z}$ denoting the ring of integers) of forms $(\theta, \omega)$ on $X$ consists of (1) a smooth (i.e., $\left.C^{\infty}\right)(r-1)$-form $\omega$ defined on $X$ except perhaps for a smooth polyhedron $e(\omega)$ of dimension $\leqq n-r$, (2) a smooth $r$-form $\theta$ defined on $X$ except for a subpolyhedron $e(\theta)$ of $e(\omega)$ of dimension $\leqq n-r-1$; we require that (3) $d \omega=\theta$ in $X-e(\omega)$, and (4) for any smooth admissible integral chain $c$ (i.e., such that $|c| \cap e(\theta)=\varnothing,|\partial c| \cap e(\omega)=\varnothing$, where $|a|$ denotes the support of the chain $a$ ), the residue relative to $c$

$$
h(\theta, \omega) \cdot c=\int_{c} \theta-\int_{\partial_{c}} \omega
$$

is an integer. We say that two $(\boldsymbol{Z}, r)$-pairs $(\theta, \omega)$ and $\left(\theta^{\prime}, \omega^{\prime}\right)$ are equivalent if $h(\theta, \omega) \cdot c=h\left(\theta^{\prime}, \omega^{\prime}\right) \cdot c$ whenever both members are defined. The equivalence classes of $(\boldsymbol{Z}, r)$-pairs constitute a differential graded module $\mathfrak{\complement} r(X, \boldsymbol{Z})$ with exterior differential defined by $d(\theta, \omega)=(0, \theta)$. The derived cohomology module $\mathfrak{S}^{*}(X, \boldsymbol{Z})$ has anti-commutative algebra structure, and equation (1) determines a canonical isomorphism of $\mathfrak{S}^{*}(X, Z)$ onto the singular cohomology algebra $H^{*}(X, \boldsymbol{Z})$ of $X$. The same theorem is valid with $\boldsymbol{Z}$ replaced by $\boldsymbol{Z}_{2}$ (the integers modulo 2 ), if we require that equivalent $(\boldsymbol{Z}, r)$-pairs have the same residues mod 2 .

(B) Now suppose that $X$ is orientable and has a Riemann structure. For every $r(1 \leqq r \leqq n)$ we define the $r$ th Gauss curvature form $\Omega^{(r)}$ and the $r$ th geodesic curvature form $\Phi^{(r)}$ of $X$. These are smooth forms on the bundle $\delta_{n-r}(X)$ of orthonormal $(n-r)$-frames on $X$ (we define $\left.\delta^{0}(X)=X\right)$; these forms are intrinsically associated with the Riemann structure of $X$. Furthermore, if $V$ is an $r$-dimensional submanifold, then $\Omega^{(r)}$ and $\Phi^{(r-1)}$ determine the Gauss and geodesic curvature forms of $V$ (relative to its induced Rie-

Presented to the Society, January 30, 1958; received by the editors December 21, 1957.

(1) Research partially supported by the Office of Naval Research. 
mann structure). Actually, $\Phi^{(r-1)}$ has opposite sign to the geodesic curvature form of Chern [4]; our choice is dictated by the desire to fit equation (2) below into our general residue theory.

Take any smooth $(n-r+1)$-frame $f_{r-1}(x)=\left(x ; e_{1} \cdots e_{n-r+1}\right)$ defined on $X$ except perhaps for a smooth $(n-r)$-polyhedron $e\left(f_{r-1}\right)$. Let $f_{r}$ be a smooth extension of $\left(x ; e_{1} \cdots e_{n-r}\right)$, defined on $X$ except for a smooth $(n-r-1)$ subpolyhedron of $e\left(f_{r-1}\right)$. We will show that $\left(f_{r}^{*} \Omega^{(r)}, f_{r-1}^{*} \Phi^{(r-1)}\right)$ is a $(Z, r)$-pair on $X$, and

$$
w^{r}(X) \cdot c=\int_{c} f_{r}^{*} \Omega^{(r)}-\int_{\partial_{c}} f_{r-1}^{*} \Phi^{(r-1)}
$$

for any integral $r$-chain admissible for the pair. Here and henceforth (2) is to be considered a congruence modulo 2 if $r$ is even and $<n$. In case $r=n$ equation (2) contains the Gauss-Bonnet Theorem for closed orientable Riemann manifolds (see Allendoerfer-Weil [1] and Chern [4]), because of the relation $w^{n}(X) \cdot X=\chi(X)$, the Euler characteristic of $X$. It is known (Theorem of Thom) that the Stiefel-Whitney classes are topological invariants of $X$; formula (2) relates them to invariants of its Riemann structure.

These integral formulas for $w^{r}(X)$, in a different form and derived by different methods, have been discovered by Allendoerfer [2], using forms on the $(n-r)$-skeleton of a particular cellular subdivision of $X$ (and expressed in polar coordinates defined in each cell of that skeleton). Our formulas (2) should be considered as reinterpreting Allendoerfer's, emphasizing the geometric role played by the forms $\Omega^{(r)}$ and $\Phi^{(r-1)}$.

Also, the referee has called attention to a paper of Chern, Integral formulas for the characteristic classes of sphere bundles, Proc. Nat. Acad. Sci. vol. 30 (1944) pp. 269-273. In that work a closed transgressive form (apparently different (for $r<n$ ) from our $\Phi^{(r-1)}$ ) is constructed on $S_{n-r+1}(X)$ for $r$ odd, and its relation to the $r$ th Stiefel-Whitney class of $X$ is studied by integration over bounded $(r-1)$-submanifolds of $X$.

Formula (2) can be derived in two distinct ways: (1) by considering $X$ as an abstract manifold and studying the intrinsic geometry of its frame bundles, and (2) by imbedding $X$ in Euclidean space (of sufficiently high dimension) and considering the geometry of $X$ induced from the classifying space - the Grassmann manifold $G_{n+m, n}$ of oriented $n$-planes in Euclidean $(n+m)$-space. Considering that second point of view we obtain (Theorem 3C) a set of partially invariant $(\boldsymbol{Z}, r)$-pairs of forms on $G_{n+m, n}$ forming a base for $H^{*}\left(G_{n+m, n}, \boldsymbol{Z}_{2}\right)$. I hope to present elsewhere a detailed study of invariant $(\boldsymbol{Z}, r)$-pairs of forms on other homogeneous spaces, having orbital singularities.

Added in proof. It has been brought to my attention that the formula (2) and the geometric interpretation of the forms $\left(\Omega^{(r)}, \Phi^{(r-1)}\right)$ have been discovered by S. Takizawa, Mem. Coll. Sci. Univ. Kyoto vol. 28 (1953) pp. 1-10 
and pp. 241-251. See also A. Aragnol, C.R. Acad. Sci. Paris vol. 238 (1954) pp. 2387-2389. Both authors use the method of Allendoerfer.

2. Universal curvature forms. (A) Let $E_{n+m}$ denote Euclidean $(n+m)$ dimensional space with a fixed orthonormal base $e_{1}, \cdots, e_{n+m}$; write the coordinates of a point $x \in E_{n+m}$ as $x=\left(x_{1}, \cdots, x_{n+m}\right) . E_{n+m}^{r}$ is the subspace spanned by the last $r$ vectors. Let $R_{n+m}$ denote the rotation group in $E_{n+m}$ and $R_{n+m}^{r}$ the subgroup acting in $E_{n+m}^{r}$ keeping $x_{i}, \cdots, x_{n+m-r}$ fixed.

For each $r(1 \leqq r \leqq n)$ let $V_{n+m, n}^{r}$ denote the pairs $(X, f)$, where $X$ is an oriented $n$-plane through the origin 0 in $E_{n+m}$ and $f$ is an $(n-r)$-frame at 0 in $X$; we agree that $V_{n+m, n}^{n}$ is the Grassmann manifold $G_{n+m, n}$ of oriented $n$ planes through the origin in $E_{n+m}$. Each $V_{n+m, n}^{r}$ is expressible as the factor space $R_{n+m} / R_{r} \times R^{m}$, and $V_{n+m, n}^{1}$ is naturally identified with the Stiefel manifold $V_{n+m, n}$ of $n$-frames at 0 in $E_{n+m}$. The coset maps define a sequence of $R_{n}$-bundles

$$
V_{n+m, n}=V_{n+m, n}^{1} \rightarrow \cdots \rightarrow V_{n+m, n}^{r} \rightarrow \cdots \rightarrow V_{n+m, n}^{n}=G_{n+m, n} .
$$

Each composition map $V_{n+m, n}^{r} \rightarrow G_{n+m, n}$ is an associated $R_{n}$-bundle of the principal bundle $V_{n+m, n} \rightarrow G_{n+m, n}$, with fibre $V_{n, n-r}=R_{n} / R_{r}$.

(B) Maurer-Cartan forms for $R_{n+m}$ can be taken as the entries $\omega_{i j}$ $(1 \leqq i, j \leqq n+m)$ in the skew-symmetric matrix $w=u^{-1} d u$ for any $u \in R_{n+m}$, using the base in $E_{n+m}$. Let $L^{*}\left(R_{n+m}, R_{r} \times R^{m}\right)$ denote the (real) linear space of left-invariant 1 -forms on $R_{n+m}$ which are zero on the Lie algebra $L\left(R_{r} \times R^{m}\right)$; we observe that for any $r(1 \leqq r \leqq n)$ the space $L^{*}\left(R_{n+m}, R_{r} \times R^{m}\right)$ is spanned by the $\omega_{i j}$ such that $(1 \leqq i \leqq n, r+1 \leqq j \leqq n+m)$.

We will say that a left invariant form $\alpha$ (of any degree) on $R_{n+m}$ is $V_{n+m, n}^{r}$-basic $(1 \leqq r \leqq n)$ if there is a form $\beta$ on $V_{n+m, n}^{r}$ such that $\pi^{*} \beta=\alpha$, where $\pi$ is the coset map $R_{n+m} \rightarrow V_{n+m, n}^{r} ; \pi^{*}$ is a monomorphism because the fibering is locally trivial. $\beta$ is then invariant under the action of $R_{n+m}$ on $V_{n+m, n}^{r}$. It is well known that the following is a pair of necessary and sufficient conditions that $\alpha$ be $V_{n+m, n}^{r}$-basic: (1) $\alpha$ is in the exterior subalgebra generated by $L^{*}\left(R_{n+m}, R_{r} \times R^{m}\right)$, and (2) $\alpha$ is invariant under the adjoint action of $\operatorname{ad}\left(R_{r} \times R^{m}\right)$. If $\alpha$ is $V_{n+m, n}^{r}$-basic then we will treat $\alpha$ as though it were in fact defined on $V_{n+m, n}^{r}$.

For any $v \in R_{n+m}$ we have $(\mathrm{ad} v) w=v^{-1} w v$; the following computations are elementary:

(1) If $1 \leqq i \leqq n, 1 \leqq j \leqq n$, then

$$
\begin{aligned}
& (\operatorname{ad} v) \omega_{i j}=\omega_{i j} \quad \text { if } v \in R^{m} ; \\
& (\operatorname{ad} v) \omega_{i j}=\sum_{p, q=1}^{n} v_{p i} \omega_{p q} v_{q j} \quad \text { f } v \in R_{n} .
\end{aligned}
$$

(2) If $1 \leqq i \leqq n, n+1 \leqq j \leqq n+m$, then 


$$
\begin{array}{ll}
(\operatorname{ad} v) \omega_{i j}=\sum_{k=n+1}^{n+m} \omega_{i k} v_{k j} & \text { if } v \in R^{m}, \\
(\operatorname{ad} v) \omega_{i j}=\sum_{k=1}^{n} v_{k i} \omega_{k j} & \text { if } v \in R_{n} .
\end{array}
$$

(C) Definition. The $(r, n)$-universal curvature matrix is the matrix of 2 -forms on $R_{n+m}$ whose entries are the exterior polynomials

$$
\Omega_{i j}^{(r)}=\sum_{k=r+1}^{n+m} \omega_{i k} \bigvee \omega_{k j}, \quad(1 \leqq i, j \leqq n)
$$

where $\bigvee$ denotes exterior multiplication. The $(r, n)$-universal Gauss curvature form

$$
\begin{aligned}
\Omega^{(r)} & =\frac{(-1)^{r / 2}}{2^{r} \pi^{r / 2}(r / 2) !} \sum \epsilon_{i_{1}} \cdots i_{r} \Omega_{i_{1} i_{2}}^{(r)} \vee \cdots \vee \Omega_{i_{r-1} i_{r}}^{(r)} & & \text { if } r \text { is even, } \\
& =0 & & \text { if } r \text { is odd }
\end{aligned}
$$

the summation is taken over all selections $\left(i_{1} \cdots i_{r}\right)$ from $(1 \cdots n)$. We will see in $\$ 4$ that the $\Omega_{i j}^{(r)}$ determine the curvature matrix for every $r$-dimensional submanifold of a Riemann $n$-dimensional submanifold of $E_{n+m}$.

It is well known (and easily seen) that the forms $\omega_{i j}(1 \leqq i, j \leqq n)$ are $V_{n+m, n}$-basic and are the forms of an affine connection for the principal $R_{n}$-bundle $V_{n+m, n} \rightarrow G_{n+m, n}$; its curvature matrix has the entires (3) with $r=n$. Furthermore, $\Omega^{(n)}$ is $G_{n+m, n}$-basic and is the universal Gauss-Bonnet form; see Chern [6].

Consider now the corresponding situation for the associated bundles.

Lemma. $\Omega^{(r)}$ is $V_{n+m, n}^{r}$-basic; thus it can be considered as an $R_{n+m}$-invariant form on $V_{n+m, n}^{r}$.

Proof. Clearly $\Omega^{(r)}$ is in the subalgebra generated by $L^{*}\left(R_{n+m}, R_{r} \times R^{m}\right)$. Applying (1) and (2) we see that $(\operatorname{ad} v) \Omega_{i j}^{(r)}=\Omega_{i j}^{(r)}$ for any $v \in R^{m}$, whence $(\operatorname{ad} v) \Omega^{(r)}=\Omega^{(r)}$. Similarly, if $v \in R_{r}$ then

$$
(\operatorname{ad} v) \Omega_{i j}^{(r)}=\sum_{p, q=1}^{n} v_{p i} v_{q j} \Omega_{p q}^{(r)}
$$

the determinant identity $\epsilon_{i_{1}} \cdots i_{r} v_{p_{1} i_{1}} \cdots v_{p_{r} i_{r}}=\epsilon_{p_{1}} \cdots p_{r}$ for $v \in R_{r}$ shows that $(\operatorname{ad} v) \Omega^{(r)}=\Omega^{(r)}$. The lemma now follows from the result quoted in (B).

(D) A key step in Chern's proof [4] of the Gauss-Bonnet Theorem shows that the curvature form $\Omega$ of an orientable Riemann manifold $X$ is derived in the tangent sphere bundle $\pi: \delta(X) \rightarrow X$; i.e., there is an $(r-1)$-form (the geodesic curvature form) $\Phi$ on $\delta(X)$ such that $\pi^{*} \Omega=d \Phi$. (As remarked in our Introduction, Chern actually uses $-\Phi$.) Furthermore, the restriction of $\Phi$ to a fibre $S_{n-1}$ of $\mathrm{S}(X)$ determines (in the sense of de Rham's Theorem) a gen- 
erator for $H^{n-1}\left(S_{n-1}, \boldsymbol{Z}\right)$. These properties are sometimes expressed by saying that $(\Omega, \Phi)$ is a transgressive pair of forms in the sphere bundle of $X$. Chern's method also gives a universally transgressive pair $\left(\Omega^{(n)}, \Phi^{(n-1)}\right)$ in the sphere bundle $V_{n+m, n}^{n-1} \rightarrow G_{n+m, n}$. We now show that a similar result (with the same type of construction) holds for every $r(2 \leqq r \leqq n)$.

If $[r / 2]$ denotes the largest integer $\leqq r / 2$, then for all $k(0 \leqq k \leqq[r / 2]-1)$ we define the exterior polynomials on $R_{n+m}$

$$
\begin{aligned}
& \Phi_{k}^{(r-1)}=\sum \epsilon_{i_{1}} \cdots i_{r-1} \Omega_{i_{1} i_{2}}^{(r)} \bigvee \cdots \vee \Omega_{i_{2 k-1} i_{2 k}}^{(r)} \bigvee \omega_{i_{2 k+1} r} \bigvee \cdots \vee \omega_{i_{r-1} r}, \\
& \Psi_{k}^{(r-1)}=2(k+1) \sum \epsilon_{i_{1}} \cdots i_{r-1} \Omega_{i_{1} i_{2}}^{(r)} \bigvee \cdots \vee \Omega_{i_{2 k+1} r}^{(r)} \bigvee \omega_{i_{2 k+2} r} \bigvee \cdots \vee \omega_{i_{r-1} r} \cdot
\end{aligned}
$$

Set $\Psi_{-1}^{(r-1)}=\Psi_{[r / 2]}^{(r-1)}=0$, and observe that $\Phi_{[r-2]}^{(r-1)}$ is defined if $r$ is odd. (Superscripts do not necessarily denote degrees!)

LEMmA. If $2 \leqq r \leqq n$ and $0 \leqq k \leqq[r / 2]-1$, then the forms $\Phi_{k}^{(r-1)}$ and $\Psi_{k}^{(r-1)}$ are $V_{n+m, n}^{r-1}$-basic and satisfy

$$
d \Phi_{k}^{(r-1)}=\Psi_{k-1}^{(r-1)}+\frac{r-2 k-1}{2(k+1)} \Psi_{k}^{(r-1)} .
$$

The proof that the forms are basic is just like that of Lemma $2 \mathrm{C}$. The proof of (5)-computed in $V_{n+m, n}$-follows Chern's proof [4] (see also Flanders [7] for a detailed exposition using vector valued forms) for the case $r=n$.

Set

$$
a_{k}=\frac{(-1)^{r+k-1}}{2^{r} \pi^{(r-1) / 2} k ! \Gamma\left(\frac{r-2 k+1}{2}\right)}
$$

and

$$
\Phi^{(r-1)}=\sum_{k=0}^{[(r-1) / 2]} a_{k} \Phi_{k}^{(r-1)}
$$$$
\text { for } 2 \leqq r \leqq n
$$

Then $\Phi^{(r-1)}$ is an $R_{n+m}$-invariant form on $V_{n+m, n}^{r-1}$, which we call the $(r-1, n)$ universal geodesic curvature form; by combining (5) and (7) we obtain

$$
d \Phi^{(r-1)}=\Omega^{(r)} .
$$

In particular, $\Phi^{(r-1)}$ is closed if $r$ is odd.

The bundle $V_{n+m, n}^{r-1} \rightarrow V_{n+m, n}^{r}$ has typical fibre $S_{r-1}=R_{r} / R_{r-1}$. The restriction of $\Phi^{(r-1)}$ to $S_{r-1}$ is $a_{0} \Phi_{0}^{(r-1)}$, which determines a generator of $H^{r-1}\left(S_{r-1}, Z\right)$. We summarize these results in the

Proposition. Considered as $R_{n+m}$-invariant forms on $V_{n+m, n}^{r}$ and $V_{n+m, n}^{r-1}$ respectively, $\left(\Omega^{(r)}, \Phi^{(r-1)}\right)$ is a transgressive pair in the $(r-1)$-sphere bundle $V_{n+m, n}^{r-1} \rightarrow V_{n+m, n}^{r}$. 
3. $R_{n}$-universal Stiefel-Whitney classes. (A) We will suppose henceforth that $m \geqq n+1$. Any sequence of linear subspaces $0 \subset L_{1} \subset \cdots \subset L_{n+m}=E_{n+m}$ (with subscripts denoting dimensions; for exposition see Chern [5] or Wu [10]) determines an oriented cellular subdivision of the Grassmann manifold $G_{n+m, n}$, defined in terms of the Schubert varieties $\left(a_{1} \cdots a_{n}\right)=\left\{X \in G_{n+m, n}\right.$ : $\left.\operatorname{dim}\left(X \cap L_{a_{i+i}}\right) \geqq i,(i \leqq i \leqq n)\right\}$, where the $a_{i}$ are integers satisfying $0 \leqq a_{1}$ $\leqq \cdots \leqq a_{n} \leqq m$. Each $\left(a_{1} \cdots a_{n}\right)$ is a circuit (pseudomanifold) of dimension $a_{1}+\cdots+a_{n}$, expressible as the closure of the union of two open oriented cells $\left(a_{1} \cdots a_{n}\right)^{+}$and $\left(a_{1} \cdots a_{n}\right)^{-}$. We will let $\left(a_{1} \cdots a_{n}\right)^{ \pm}$also stand for the elementary cochain (with integer or integer mod 2 coefficients) which is +1 on the chain $\left(a_{1} \cdots a_{n}\right)^{ \pm}$and zero on other cells.

For each $r(1 \leqq r \leqq n)$ let $w_{r}$ denote the chain

$$
w_{r}=(0 \cdots 0 \underset{\leftarrow r \rightarrow}{1} \cdots)^{+}-(0 \cdots 0 \underset{\leftarrow r \rightarrow}{1 \cdots})^{-},
$$

and let $w^{r}$ denote the corresponding cochain. It follows from general boundary-coboundary formulas for Schubert varieties that

$$
\partial w_{r}=\left[1+(-1)^{r+1}\right] w_{r-1}, \quad d w^{r}=\left[1+(-1)^{r}\right] w^{r+1} .
$$

In particular, $w^{r}$ is a cocycle if $r$ is odd or $r=n$, and $w^{r}$ is a cocycle mod 2 if $r$ is even.

The $R_{n}$-universal Stiefel-Whitney classes are the cohomology classes $\left(w^{r}\right) \in H^{r}\left(G_{n+m, n}, \boldsymbol{Z}\right)$ of $w^{r}$ if $r$ is odd or $r=n$, and $\left(w^{r}\right) \in H^{r}\left(G_{n+m, n}, Z_{2}\right)$ if $r$ is even and $r<n$. These classes are independent of the particular sequence of linear subspaces chosen to define the subdivision and of $m \geqq n+1$. The class $\left(w^{n}\right)$ is of infinite order and is sometimes called the Euler-Poincare class. The other Stiefel-Whitney classes have order 2.

REMARK. Let $x^{r-1}$ denote a generator of $H^{r-1}\left(V_{n, n-r+1}, Z\right)$ if $r$ is odd or $r=n$, and of $H^{r-1}\left(V_{n, n-r+1}, Z_{2}\right)$ if $r$ is even and $r<n$. Since $V_{n, n-r+1}$ is $(r-2)$ connected it follows that $x^{r-1}$ is transgressive in the bundle $V_{n+m, n}^{r-1} \rightarrow G_{n+m, n}$. Using the technique in (C) below Pontrjagin has shown that the unique transgressive image of $x^{r-1}$ is the rth universal Stiefel-Whitney class.

(B) Let us consider the oriented subdivision $K$ of $G_{n+m, n}$ defined by the linear spaces

$$
\begin{aligned}
L_{1} & =\left\{x=\left(0, \cdots, 0, x_{n+1}, 0, \cdots, 0\right)\right\}, \\
L_{2} & =\left\{x=\left(0, \cdots, 0, x_{n}, x_{n+1}, 0 \cdots 0\right)\right\}, \cdots, \\
L_{n+2} & =\left\{x=\left(x_{1}, \cdots, x_{n+2}, 0, \cdots, 0\right)\right\}, \cdots, L_{n+m}=E_{n+m} .
\end{aligned}
$$

If in the definition of Schubert varieties we replace $L_{a_{i+i}}$ by $L^{a_{i}+i}$ (letting $L^{p}$ denote the oriented orthogonal complement of $L_{n+m-p}$ ), we obtain an oriented dual subdivision $K_{*}$ of $G_{n+m, n}$. Using stars to indicate the cells of $K_{*}$, the dual $(n m-r)$-cycle (or cycle mod 2) D $w^{r}$ of $w^{r}$ is given by 


$$
\begin{aligned}
D w^{r}=(-1)^{\lambda}\left[(m-1 \cdots m-1 m \cdots m)_{*}^{+}\right. & \\
& \left.-(-1)^{n+m}(m-1 \cdots m-1 m \cdot m m)_{*}^{-}\right]
\end{aligned}
$$

if $n(n-1) / 2$ is even, and

$$
\begin{aligned}
=(-1)^{\lambda+1}\left[(-1)^{n+m}(m-1 \cdots m-1 m \cdots m)_{*}^{+}\right. & \\
& \left.-(m-1 \cdots m-1 m \cdots m)_{*}^{-}\right]
\end{aligned}
$$

if $n(n-1) / 2$ is odd, where $(-1)^{\lambda}$ is the algebraic number of intersections of the dual cells $(0 \cdots 01 \cdots 1)^{+}(r$ ones $)$ and $(m-1 \cdots m-1 m \cdots m)_{*}^{+}$; see $\mathrm{Wu}[10$, Chapter I, p.3].

(C) Definition. Relative to the dual subdivisions $K$ and $K_{*}$ of $G_{n+m, n}$ described in (B) we define an admissible pair of sections

$$
\begin{gathered}
f_{p} ; G_{n+m, n}-(m-1 \cdots m-1 m \cdots m)_{*} \rightarrow V_{n+m, n}^{p} \\
\longleftarrow p+1 \longrightarrow
\end{gathered}
$$

as a pair of smooth sections $(p=r-1, r)$ such that $\pi \circ f_{r-1}=f_{r}$, where $\pi$ is the bundle map $\pi: V_{n+m, n}^{r-1} \rightarrow V_{n+m, n}^{r}$. Admissible pairs can be constructed for all $r$ following a method of Pontrjagin $[8, \S 1 \mathrm{D}]$; see also Chern $[5$, p. 93]. We will suppose that $2 \leqq r \leqq n$; the case $r=1$ is trivial.

Theorem. Let $\Omega^{(r)}$ and $\Phi^{(r)}$ denote the $(r, n)$-universal Gauss and geodesic curvature forms; set

$$
\omega=f_{r}^{*} \Omega^{(r)} \quad \text { and } \quad \phi=f_{r-1}^{*} \Phi^{(r-1)} .
$$

Then $(\omega, \phi)$ is a $(\boldsymbol{Z}, r)$-pair $(2 \leqq r \leqq n)$ on $G_{n+m, n}$ whose singularities are the supports of $\partial \mathrm{Dw}^{r}$ and $D w^{r}$ respectively. For any integral $r$-chain $a_{r}$ of $K$ we have

$$
w^{r} \cdot a_{r}=\int_{a_{r}} \omega-\int_{\partial a_{r}} \phi .
$$

In particular, the residues of $(\omega, \phi)$ are independent of the choice of admissible pair of sections used to define them.

Proof. The statement about the singularities follows immediately from (B). In its domain of definition we have $d f_{r-1}^{*} \Phi^{(r-1)}=f_{r-1}^{*} \circ \pi^{*} \Omega^{(r)}=f_{r}^{*} \Omega^{(r)}$, and therefore $d \phi=\omega$. To show that the pair has integral residues (on all admissible integral chains) it suffices to verify (3) for the integral $r$-chains of $K$, by Allendoerfer-Eells [3, Corollary 5A].

We see that $w^{r} \cdot a_{r}$ is zero on the cells of $K$ except when $a_{r}$ is $\sigma_{r}( \pm)$ $=\left(\begin{array}{llllll}0 & \cdots & 1 & \cdots & 1 & \pm\end{array}\right)^{ \pm}(r$ ones $)$, in which case $w^{r} \cdot \sigma_{r}( \pm)= \pm 1$. On the other hand, if $a_{r} \neq \sigma_{r}( \pm)$ then $a_{r}$ and $D w^{r}$ do not intersect, whence we can apply Stokes' Theorem to conclude that the right member of (3) is zero; otherwise we find that 


$$
\begin{array}{rlrl}
\int_{\sigma_{r}( \pm)} \omega-\int_{\partial \sigma_{r}( \pm)} \phi & =\int_{\sigma_{r}( \pm)} \omega+\int_{\sigma_{r-1}( \pm)} \phi+\int_{\sigma_{r-1}(\mp)} \phi & & \text { if } r \text { is even, and } \\
& =-\int_{\sigma_{r-1}( \pm)} \phi+\int_{\sigma_{r-1}(\mp)} \phi & \text { if } r \text { is odd. }
\end{array}
$$

Now the Schubert variety $(0 \cdots 01 \cdots 1)=\left\{X \in G_{n+m, n}: L_{n-r} \subset X \subset L_{n+1}\right\}$ is naturally identified with the unit $r$-sphere in $E_{r+1}$, and $\sigma_{r}( \pm)$ are complementary oriented hemispheres. By considering the restrictions of (4) and (7) of $\S 2$ to the parts of the associated bundles $V_{n+m, n}^{p}$ over the integration domains of (4), we see that on those domains $\omega$ and $\phi$ are, independently of the choice of the admissible pair of sections,

and

$$
\frac{\Gamma\left(\frac{r+1}{2}\right)}{\pi^{(r+1) / 2}} \omega_{1, r+1} \vee \cdots \vee \omega_{r, r+1}
$$

$$
\frac{\Gamma\left(\frac{r}{2}\right)}{2 \pi^{r / 2}} \omega_{1, r} \vee \cdots \vee \omega_{r-1, r}
$$

Considering the orientation conventions (Wu $[10, \mathrm{p} .17]$ ) for the cells of $K$, we find that the right members of (4) equal one. Thus (3) is established in every case, and the proof is complete. Compare this proof with that of Chern's representation formula $[5$, p. 77] for the universal Chern classes.

By the isomorphism theorem quoted in $\$ 1 \mathrm{~A}$ we obtain the

CoROllary. The cohomology class with $\boldsymbol{Z}$ (resp. $\boldsymbol{Z}_{2}$ ) coefficients if $r$ is odd or $r=n$ (resp., $r$ is even and $<n)$ of the pair $(\omega, \phi)$ corresponds to the rth universal Stiefel-Whitney class.

(D) We consider briefly a dual situation. For each $r(1 \leqq r \leqq m)$ let $\bar{V}_{n+m, m}^{r}=R_{n+m} / R_{n} \times R_{r}^{m}$; each $\bar{V}_{n+m, m}^{r} \rightarrow G_{n+m, n}$ is an associated bundle of the principal $R^{m}$-bundle $\bar{V}_{n+m, m} \rightarrow G_{n+m, n}$ with fibre $V_{m, m-r}$. If we change the order of the base in $E_{n+m}$, setting $e_{i}^{\prime}=e_{n+m-i+1}$, and let $R_{r}^{\prime}$ denote the rotation group in the variables $x_{1}^{\prime}, \cdots, x_{r}^{\prime}$, etc., then the natural involutory homeomorphism $\alpha: G_{n+m, n} \rightarrow G_{n+m, m}^{\prime}$ induces the bundle $\bar{V}_{n+m, m}^{r} \rightarrow G_{n+m, n}$ from $V_{n+m, m}^{\prime r} \rightarrow G_{n+m, m}^{\prime}$.

The Maurer-Cartan forms $\bar{\omega}_{i j}=\omega_{n+m-i+1, n+m-j+1}(1 \leqq i, j \leqq m)$ are $\bar{V}_{n+m, m}$ basic and define an invariant connection in the $R^{m}$-bundle $\bar{V}_{n+m, m} \rightarrow G_{n+m, n}$. We define the dual $(r, m)$-universal curvature matrix as the matrix of 2 -forms on $R_{n+m}$ whose entries are

$$
\bar{\Omega}_{i j}^{(r)}=\sum_{k=r+1}^{n+m} \bar{\omega}_{i k} \bigvee \bar{\omega}_{k j} \quad(1 \leqq i, j \leqq m) .
$$


The dual $(r, m)$-universal Gauss and geodesic curvature forms $\bar{\Omega}^{(r)}$ and $\bar{\Phi}^{(r)}$ are defined by (4) and (7) of $\S 2$, using $\bar{\Omega}_{i j}^{(r)}$ and $\bar{\omega}_{i r}$ (and summations from 1 to $m)$. As in Proposition 2D we find that $\bar{\Omega}^{(r)}$ and $\bar{\Phi}^{(r-1)}$ are a transgressive pair in the $(r-1)$-sphere bundle $\bar{V}_{n+m, m}^{r-1} \rightarrow \bar{V}_{n+m, m}^{r}$.

Relative to a given subdivision $K$ of $G_{n+m, n}$ we define the chain $\bar{w}_{r}$ $=(0 \cdots 0 r)^{+}-(0 \cdots 0 r)^{-}$for $1 \leqq r \leqq m$; if $\bar{w}^{r}$ denotes the corresponding cochain, then $d \bar{w}^{r}=\left[1+(-1)^{r}\right] \bar{w}^{r+1}$. The dual $R_{n}$-universal Stiefel-Whitney classes are the cohomology classes $\left(\bar{w}^{r}\right) \in H^{r}\left(G_{n+m, n}, \boldsymbol{Z}\right)$ if $r$ is odd or $r=m$, and $\left(\bar{w}^{r}\right) \in H^{r}\left(G_{n+m, n}, \boldsymbol{Z}_{2}\right)$ if $r$ is even and $r<m . \bar{w}^{m}$ has infinite order, and $\bar{w}^{r}$ has order 2 for $r<m$.

We define a dual admissible pair of sections $\bar{f}_{p}: G_{n+m, n}-(m \cdots m m-p+1)_{*}$ $\rightarrow \bar{V}_{n+m, m}^{p}$ for $p=r-1, r$ as in Definition $3 \mathrm{C}$; then $(m \cdots m m-r)_{*}$ is the support of $D \bar{w}^{r}$. As dual to Theorem 3C we have the

Theorem. Let $\bar{\Omega}^{(r)}$ and $\bar{\Phi}^{(r)}$ denote the dual $(r, m)$-universal Gauss and geodesic curvature forms; for a dual admissible pair of sections $\bar{f}_{p}$ let

$$
\bar{\omega}=\bar{f}_{r}^{*} \bar{\Omega}^{(r)} \quad \text { and } \quad \bar{\phi}=f_{r-1}^{*} \bar{\Phi}^{(r-1)} .
$$

Then $(\bar{\omega}, \bar{\phi})$ is a $(\boldsymbol{Z}, r)$-pair $(1 \leqq r \leqq m)$ on $G_{n+m, n}$ representing the dual StiefelWhitney class $\bar{w}^{r}$.

4. The tangent classes. (A) Suppose that $Y$ is a smooth orientable Riemann $(n+m)$-manifold and $X$ is an orientable submanifold of dimension $n$ regularly embedded in $Y$. The connection forms $\omega_{i j}(1 \leqq i, j \leqq n+m)$ of $Y$ and its curvature forms $\Omega_{i j}$, forms defined on the bundle $S_{n+m}(Y)$ of orthonormal $(n+m)$-frames on $Y$, are entries in skew-symmetric matrices, related by

$$
d \omega_{i j}=\Omega_{i j}+\sum_{k=1}^{n+m} \omega_{i k} \bigvee \omega_{k j} \quad(1 \leqq 1, j \leqq n+m) .
$$

Consider the subbundle $\Theta_{n+m}(X)$ of $S_{n+m}(Y)$ consisting of those frames $\left(x ; e_{1} \cdots e_{n+m}\right)$ such that $x \in X$ and $e_{1}, \cdots, e_{n}$ are in the tangent space $X(x)$ of $X$ at $x$. If $f$ denotes the inclusion mapping of these bundles, then the Riemann geometry on $X$ induced from that of $Y$ has connection forms $\theta_{i j}=f^{*} \omega_{i j}$ and curvature forms

$$
\Theta_{i j}=\Omega_{i j}+\sum_{k=n+1}^{n+m} \omega_{i k} \bigvee \omega_{k j} \quad(1 \leqq i, j \leqq n)
$$

related by

$$
d \theta_{i j}=\Theta_{i j}+\sum_{k=1}^{n} \theta_{i k} \vee \theta_{k j}
$$

(B) Now let $Y=E_{n+m}(m \geqq n+1)$, and fix an orientation of $X$. Let $T: X \rightarrow G_{n+m . n}$ be the tangent map, defined by letting $T(x)$ be that oriented $n$-plane in $G_{n+m, n}$ which is parallel to the tangent space $X(x)$ and has the 
same orientation. If $T^{*}$ denotes the induced homomorphism of cohomology rings, there the tangent Stiefel-Whitney classes $w^{r}(X)$ of $X$ are defined as $T^{*}\left(w^{r}\right)$ for $1 \leqq r \leqq n$.

REMARK. It is known that $w^{1}(X)=0$ if and only if $X$ is orientable. By using forms with twisted coefficients we can prove an analogue of Theorem $4 \mathrm{D}$ below, deleting the hypothesis that $X$ is orientable, and obtain an integral representation of $w^{1}(X)$. That formula expresses the elementary fact that $X$ is orientable if and only if the bundle $S_{n}(X)$ is disconnected.

Henceforth we assume that $2 \leqq r \leqq n$.

The classes $w^{r}(X)$ can be (in fact, were first) defined without reference to imbedding, in terms of transgression in the bundle $S_{n-r+1}(X)$ of $(n-r+1)$ frames on $X$; i.e., as the primary obstruction to constructing a smooth section of $s_{n-r+1}(X)$ over $X$. The equivalence of these definitions is proved by using partial sections as in $\$ 3 \mathrm{C}$ in the commutative diagram

$$
\begin{array}{cc}
S_{n-r+1}(X) & \stackrel{T_{r-1}}{\longrightarrow} V_{n+m, n}^{r-1} \\
\downarrow & \downarrow \\
X & \longrightarrow T G_{n+m, n},
\end{array}
$$

where $T_{r-1}\left(x ; e_{1} \cdots e_{n-r+1}\right)$ is the element of $V_{n+m, n}^{r-1}$ consisting of $T(x)$ and the image frame of $e_{1} \cdots e_{n-r+1}$ in $T(x)$.

(C) Definition. The rth geodesic and Gauss curvature forms of $X$ are

$$
\Phi^{(r)}(X)=T_{r}^{*} \Phi^{(r)} \quad \text { and } \quad \Omega^{(r)}(X)=T_{r}^{*} \Omega^{(r)} .
$$

REMARK. The Riemann structure on $X$ induced from $E_{n+m}$ coincides with that induced by $T_{0}$ from the invariant connection ( $\left.\$ 2 \mathrm{C}\right)$ on $V_{n+m, n}$ using Maurer-Cartan forms. It follows easily that the forms (5) are in effect constructed by substituting $\theta_{i j}$ and

$$
\Theta_{i j}^{(r)}=\Theta_{i j}+\sum_{k=r+1}^{n} \theta_{i k} \bigvee \theta_{k j} \quad(1 \leqq i, j \leqq n)
$$

in the expressions (7) and (4) of $\S 2$. In particular, the forms in (5) are intrinsically defined using the Riemann structure of $X$. Furthermore, if $V$ is an $r$-dimensional submanifold of $X$, then (replacing $n$ by $r$ and $n+m$ by $n$ in (2)) we see that the forms in (5) induce the usual Gauss and geodesic curvature forms on $V$, using the construction in (A).

From the diagram (4) and Proposition 2D we obtain the

Proposition. If $X$ is an abstract smooth orientable Riemann manifold, then $\left(\Omega^{(r)}(X), \Phi^{(r-1)}(X)\right)$ is a transgressive pair in the $(r-1)$-sphere bundle $S_{n-r+1}(X) \rightarrow S_{n-r}(X)$.

(D) As in $\S 3 \mathrm{C}$ we make the 
Definition. An admissible $r$-pair of frames on $X$ are smooth sections

$$
f_{p}: X-e\left(f_{p}\right) \rightarrow S_{n-p}(X) \quad \text { for } p=r-1, r
$$

such that $\pi \circ f_{r-1}=f_{r}$ in their common domain $\left(\pi\right.$ is the bundle map $S_{n-r+1}(X)$ $\left.\rightarrow S_{n-r}(X)\right)$, where $e\left(f_{p}\right)$ is a smooth polyhedron of dimension $\leqq n-p-1$. It has been shown by Stiefel $[9, \S 4]$ that admissible $r$-pairs always exist such that $e\left(f_{p}\right)$ are the supports of the characteristic cycles of $X$ defined by $f_{p}$.

By an elementary computation of residues analogous to that made in Theorem $3 \mathrm{C}$ we have our main result.

THEOREM. Let $X$ be a smooth orientable Riemann $n$-manifold and let $\Omega^{(r)}(X), \Phi^{(r)}(X)$ be the rth Gauss and geodesic curvature forms of $X$. Then for any admissible $r$-pair of frames on $X,\left(f_{r}^{*} \Omega^{(r)}(X), f_{r-1}^{*} \Phi^{(r-1)}(X)\right)$ is a $(\boldsymbol{Z}, \boldsymbol{r})$-pair on $X$ whose cohomology class is the rth Stiefel-Whitney class of $X(2 \leqq r \leqq n)$; i.e., for every smooth integral $r$-chain $c$ such that $|c| \cap e\left(f_{r}\right)=\varnothing,|\partial c| \cap e\left(f_{r-1}\right)$ $=\varnothing$, we have

$$
w^{r}(X) \cdot c=\int_{c} f_{r}^{*} \Omega^{(r)}(X)-\int_{\partial c} f_{r-1}^{*} \Phi^{(r-1)}(X) .
$$

Equation (6) is considered a congruence modulo 2 if $r$ is even and $<n$.

REMARK. Suppose again that $X$ is imbedded in $E_{n+m}$; we ask whether we can use the diagram (4) and Theorem $3 \mathrm{C}$ to construct a pair $\left(T^{*} \omega, T^{*} \phi\right)$ to be used in (6). For an arbitrary position of $X$ in $E_{n+m}$ we see that the singularities of such a pair may not have the right dimensions to satisfy the requirements for a $(\boldsymbol{Z}, r)$-pair. However, if we imbed $X$ so that $T$ is one-one, then it is easy to see that we can construct partial sections $g_{p}: X \rightarrow S_{n-p}(X)$ so that (1) $T_{p} \circ g_{p}=f_{p} \circ T$ for $p=r-1, r$, and (2) $g_{r}=\pi \circ g_{r-1}$, where the $f_{p}$ are an admissible pair of sections as in $\S 3 \mathrm{C}$. Then $g_{r}^{*} \Omega^{(r)}(X)=T^{*} \omega$ and $g_{r-1}^{*} \Phi^{(r-1)}(X)=T^{*} \phi$.

(E) Returning to the situation in (A), we can define the normal StiefelWhitney classes $\bar{w}^{r}(X, Y)(1 \leqq r \leqq m)$ of $X$ in $Y$ (e ${ }^{r}$., as the primary obstructions to constructing smooth sections of appropriate bundles of normal frames to $X$ in $Y$ ). By paralleling the development of $\S 3 \mathrm{D}$ we obtain the dual of Theorem 4D, representing $\bar{w}^{r}(X, Y)$ in terms of normal curvature forms $\bar{\Omega}^{(r)}(X, Y)$ and $\bar{\Phi}^{(r-1)}(X, Y)$. In case $m=n=r$ and $X$ is compact we obtain Chern's formula [4, p. 683] for Whitney's invariant of $X$ in $Y$.

If we prescribe a normal frame $f$ on $X$ then the same construction gives an integral representation for the relative Stiefel-Whitney classes $w^{r}(X, Y, f)$ in terms of curvature forms. These formulas generalize the form of the GaussBonnet Theorem for manifolds $Y$ with boundary $X$ (where $f$ is the inwardly drawn normal vector field); see Allendoerfer-Weil [1].

PROBLEM. In $\S \S 2,3$ we have constructed certain partially invariant exterior polynomials in Maurer-Cartan forms of the rotation group, which determine a base for the cohomology ring $(\bmod 2)$ of a classifying space for $R_{n}$; 
furthermore, these polynomials could be interpreted as forms in a sequence of bundles associated with the $R_{n}$-universal bundle. Can we define a product of these polynomial pairs (presumably in a Whitney sum of associated bundles) and thereby construct an analogue (with mod 2 coefficients) of the theory (with real coefficients) of invariant polynomials of Chern-Weil (see Chern [5, Chapter 3])?

\section{BIBLIOGRAPHY}

1. C. B. Allendoerfer and A. Weil, The Gauss-Bonnet Theorem for Riemannian polyhedra, Trans. Amer. Math. Soc. vol. 53 (1943) pp. 101-129.

2. C. B. Allendoerfer, Characteristic cohomology classes in a Riemann manifold, Ann. of Math. vol. 51 (1950) pp. 551-570.

3. C. B. Allendoerfer and J. Eells, On the cohomology of smooth manifolds. Comment. Math. Helv. vol. 32 (1958) pp. 165-179.

4. S. S. Chern, On the curvatura integral in a Riemannian manifold, Ann. of Math. vol. 46 (1945) pp. 674-684.

5. - Topics in differential geometry, mimeographed notes, Institute for Advanced Study, 1951.

6. - La gêometrie des sous-variêtés d'un espace euclidien à plusieurs dimensions, L'Enseignement Math. vol. 40 (1955) pp. 26-46.

7. H. Flanders, Development of an extended exterior differential calculus, Trans. Amer. Math. Soc. vol. 75 (1953) pp. 311-326.

8. L. S. Pontrjagin, Characteristic cycles on differentiable manifolds, Mat. Sb. vol. 21 (63) (1947) pp. 233-284; Amer. Math. Soc. Translations vol. 32 (1950).

9. E. Stiefel, Richtungsfelder und Fernparallelismus in n-dimensionalen Mannigfaltigkeiten, Comment. Math. Helv. vol. 8 (1934-1936) pp. 305-353.

10. Wu Wen-Tsun, Sur les classes caractêristiques des structures fibrées sphêriques, Actualités Sci. Ind., no. 1183, Paris, 1952.

UNiversity of CALIFornia, Berkeley, Calif. 